\section{Cahiers de Narratologie}

Analyse et théorie narratives

$16 \mid 2009$

Images et récits

\title{
In girum imus nocte et consumimur igni. L'étendard de Guy Debord
}

\section{Serge Milan}

\section{(2) OpenEdition}

\section{Journals}

Édition électronique

URL : http://journals.openedition.org/narratologie/1069

DOI : 10.4000/narratologie.1069

ISSN : $1765-307 X$

Éditeur

LIRCES

Référence électronique

Serge Milan, «In girum imus nocte et consumimur igni. L'étendard de Guy Debord », Cahiers de Narratologie [En ligne], 16 | 2009, mis en ligne le 29 mai 2009, consulté le 01 mai 2019. URL : http:// journals.openedition.org/narratologie/1069; DOI : 10.4000/narratologie.1069

Ce document a été généré automatiquement le 1 mai 2019.

\section{(c) $($ i) $(9)$}

Cahiers de Narratologie - Analyse et théorie narratives est mis à disposition selon les termes de la licence Creative Commons Attribution - Pas d'Utilisation Commerciale - Pas de Modification 4.0 International. 


\title{
In girum imus nocte et consumimur igni. L'étendard de Guy Debord
}

\author{
Serge Milan
}

C'est un beau moment, que celui où se met en mouvement

La Brigade légère, rangée en bataille derrière ses étendards, commence sa fameuse charge

un assaut contre l'ordre du monde. dans la 'Vallée de la mort', à Balaklava

Dans la notice autobiographique publiée en quatrième de couverture de la première réédition de son ouvrage le plus connu, La Société du Spectacle, Guy Debord se présente ainsi :

Guy Debord. Se disant cinéaste. Membre de l'Internationale situationniste, dont il a été l'un des fondateurs en 1957. Longtemps responsable des publications de l' IS en France. Mêlé aussi par moments à différentes activités de cette organisation dans plusieurs pays où s'est propagée l'agitation situationniste; notamment en Allemagne, Angleterre et Italie (s'étant fait appeler parfois Gondi, ou Decayeux). A publié en 1967 La Société du Spectacle. L'année suivante, a figuré parmi les meneurs du courant le plus extrémiste lors des troubles de mai 1968. À la suite de ces évènements, ses thèses ont acquis une grande influence dans l'ultra-gauchisme européen et américain. Français. Né en 1931 à Paris¹.

2 Nous souhaitions revenir sur la première phrase de cette notice, moins signalétique et plus ambiguë que la suite du texte: Guy Debord se disait cinéaste, avant même de rappeler son appartenance à l'Internationale situationniste, son œuvre principale et son rôle « lors des troubles de mai 1968 ».

3 Guy Debord a réalisé six films, dont trois qu'il définit ainsi dans un 'Aperçu chronologique' établi en 1989, encore une fois par ses soins : 
1952 Film de long métrage sans image Hurlements en faveur de Sade

1973 La Société du Spectacle réitérée sous forme d'un film de long métrage

1978 Film de long métrage In girum imus nocte et consumimur igni

4 Il faudrait ajouter à ces trois longs métrages une dernière œuvre "antitélévisuelle » réalisée pour une diffusion sur la chaîne Canal+ en janvier 1995, intitulée Guy Debord, son art et son temps, un peu plus d'un mois après sa mort, le 30 novembre 1994.

La création cinématographique de Debord semble jouir d'un statut particulier au regard de son activisme, si on la compare à son activité de photographe, de poète ou de plasticien, qui apparaît encore plus clairement si l'on se souvient des films significatifs ${ }^{2}$ et des textes sur le cinéma déjà fort nombreux des lettristes, qui ont marqué les débuts avant-gardistes de Debord à partir de 1950. La rupture entre la future Internationale Lettriste au sein de laquelle Debord s'affirme et Jean-Isidore Isou se joue en 1952, précisément autour du cinéma, et de la contestation virulente de Chaplin et de son œuvre 3. Il faut rappeler également que le premier film «sans image» de Debord en 1952, Hurlements en faveur de Sade, sera par lui considéré en $1964^{4}$ comme le volet initial d'une œuvre/manifeste Contre le cinéma, qui comprendra également deux courts métrages intitulés Sur le passage de quelques personnes à travers une assez courte unité de temps et Critique de la séparation, respectivement datant de 1959 et 1961, c'est-à-dire en pleine période d'activité de l'avant-garde situationniste, qui s'étend formellement de 1957 à 1972. Enfin on se souviendra des mots récents d'Olivier Assayas, qui lors d'un entretien sur le cinéma de Debord se demande non sans insistance si « la création de situations n'a pas à voir avec la fabrication d'un film », c'est-à-dire, précise-t-il, si « l'accomplissement au sens littéral de la théorie situationniste de la création consciente dans la vie quotidienne n'a pas à voir avec la pratique du cinéma au jour le jour sur les tournages ${ }^{5}$ ».

6 Toutefois il n'est pas sûr, à en juger par ses œuvres, que Debord aurait souscrit à cette thèse séduisante, alors que par ailleurs la vision de ses films pourrait elle aussi constituer ce qu'il appelait «la construction de situations », en ce qu'ils réfutaient, dans leur forme même, "les aventures futiles que conte le cinéma" pour "entraîner le spectateur à l'activité, en provoquant ses capacités de bouleverser sa propre vie ». Nous tâcherons de vérifier le refus de la «non-intervention » et de «l'identification du spectateur au héros » inhérentes au spectacle entendu comme aliénation narrative, selon les mots du Rapport sur la construction des situations de 1957, sorte de manifeste fondateur de $\mathrm{l}^{1} \mathrm{I} . \mathrm{S}^{6}:$ mais nous le ferons en nous intéressant précisément à son film apparemment le moins situationniste, au sens où il a été tourné en 1978, soit six ans après la dissolution de l'Internationale Situationniste, et où le récit est paradoxalement réintroduit en même temps qu'une autre forme d'identification, quitte à surgir d'un rapport inhabituel et complexe entre la succession des plans et des séquences et le commentaire off qui les accompagne, que nous tenterons d'éclaircir un tant soit peu. Il s'agit du long métrage déjà cité intitulé In girum imus nocte et consumimur igni.

Ce long métrage produit par SIMAR film, société anonyme contrôlée de fait par Gérard Lebovici, qui avait déjà produit La Société du Spectacle en 1973, a fait l'objet d'un contrat dont Debord a voulu rendre les termes publics, jusqu'à le considérer comme une œuvre littéraire à part entière ${ }^{8}$. Ce contrat lui assurait de fait une marge de manœuvre tout à fait inhabituelle, puisque

Il est entendu que l'auteur accomplira son travail en toute liberté, sans contrôle de qui que ce soit, et sans même tenir compte de quelque observation de qui que ce soit sur aucun aspect du contenu ni de la forme cinématographique qu'il lui paraîtra convenable de donner à son film. 
7 Le contrat, qui ne comporte ni ébauche de scénario ni titre provisoire, évoque seulement le «sixième film de Guy Debord », d'une durée de 90 minutes environ, en $35 \mathrm{~mm}$, noir et blanc et de langue française, comportant un certain nombre d'opérations qui se termineront « au plus tard le deuxième semestre de l'année 1978 » et pour lesquelles le producteur « déclare par les présentes s'engager à mettre à la disposition de l'auteur tous les moyens techniques et financiers afin que celui-ci puisse en assurer la réalisation ». La contrepartie de cette mise à disposition est la cession des droits d'auteurs de l'auteur au producteur, " sous réserve de l'exécution intégrale des conventions énoncées par les présentes ».

8 Le contrat prévoit également les rémunérations des techniciens (dont celle du metteur en scène) et une série de clauses techniques et financières où le statut du long métrage est assimilé avec grande insistance à celui d'une œuvre sous le contrôle "exclusif » de l'auteur, contrôle décliné en "opérations" (adaptation, dialogues, production, découpage, montage et finition) elles-mêmes spécifiées en

actions strictement limitées dans le temps pour la durée et pour le nombre de reprises, à savoir :

a) recherche de films à détourner (une ou deux fois)

b) tournage (trois ou quatre fois) chaque fois représentant environ une semaine

c) recherche d'actualités (une fois)

d) montage (une ou deux fois)

9 Ce texte, révélateur pour ce qui est de la compréhension du projet cinématographique debordien comme création aux conditions de production et de tournage tout à fait atypiques, suscite notre intérêt pour d'autres motifs. Il est exemplaire, en effet, de la profusion des paratextes à ses films voulue par Debord lui-même, qu'il s'agisse du contrat cité, d'une longue série de critiques de périodiques parues à la sortie du film, que Debord regroupe après quatre ans en un texte intitulé Ordures et décombres déballés à la sortie du film «In girum imus nocte et consumimur igni » par différentes sources autorisées ${ }^{9}$, d'une série de Notes diverses de l'auteur autour d' In girum ..., ou encore d'une "édition critique ", littéraire, de In girum imus nocte et consumimur igni publiée en 1990, sur laquelle nous reviendrons ${ }^{10}$. Limitons-nous pour l'instant à remarquer que l'exposition ostentatoire des conditions de production de l'œuvre et la multiplication de ses commentaires et de ses versions, en plus des caractéristiques formelles sur lesquelles nous allons maintenant nous arrêter brièvement, tendent à la déclasser d'un statut artistique auquel son auteur n'a par ailleurs jamais aspiré, et qu'il a même réfuté, ainsi que la notion même d'œuvre ${ }^{11}$.

Pour ce qui est du film lui-même, il s'agit de ce qu'on a pu appeler un panégyrique ${ }^{12}$, en reprenant le titre d'un texte plus tardif de Debord qui semblerait pouvoir s'appliquer en partie à ce film et à d'autres œuvres de ces années de maturité13. En fait, nous verrons que ce genre littéraire est loin de rendre compte du contenu du film en son entier, et que d'autres genres peuvent en rendre compte moins partiellement peut-être.

Mais que raconte In girum imus nocte et consumimur igni? Il est impossible de se faire une idée du film en résumant un scénario absent, et puisqu'il n'y a à proprement parler ni récit filmé, ni acteurs, ni progression dramatique au sens habituels de ces termes; il est plus aisé de résumer ce que raconte la bande son d'une part, et ce que montrent les images d'autre part, suivant cette « discrépance » qu'avait définie Jean-Isidore Isou (c'està-dire de la scission de l'alliance image/son en deux unités parallèles et autonomes).

11 La voix off de Guy Debord lui-même constitue l'essentiel de cette bande sonore : il y est question du cinéma et de son public, de la vie de Guy Debord et de l'avant-garde au sein 
de laquelle il avait lutté avec quelques amis, enfin d'un bilan de ce combat. L'une des notes « Pour l'ingénieur du son » rappelle que

Il faut égaliser partout à la même hauteur les phrases du commentaire; et autant que possible faire de même à l'intérieur de chacune de ces phrases. On ne recherche aucun effet oratoire en élevant la voix sur certains mots. Il s'agit d'obtenir un discours monotone et froid, un peu lointain ${ }^{14}$.

12 L'édition critique à laquelle nous faisions allusion est une retranscription fidèle de cette voix off debordienne monocorde et détachée, dans laquelle quelques notes de page rappellent au lecteur qu'

En 1978, un film illustrait effectivement ce discours. Il est sûr qu'un tel genre de film n'avait pas vraiment sa place dans le cinéma; comme on voit maintenant que le cinéma lui-même n'en a plus guère dans la société. Les seules paroles, à condition d'ajouter quelques notes pour aider à les comprendre, seront néanmoins instructives. Notre temps aura laissé peu d'écrits qui envisagent aussi franchement les grandes transformations qui l'ont marqué. Qu'auraient pu donc en voir et en dire de vrai ceux qui ont partagé quelque chose de ses illusions et de ses ambitions combinées ${ }^{15}$ ?

Ce «discours » ou "commentaire " accompagne des photographies, des séquences filmées par Debord et des extraits de courts et longs métrages connus ou obscurs. On verra ainsi dans ce film des publicités et clichés de la vie familiale et urbaine des classes moyennes, des portraits de groupes ou individuels, des photos d'actualités, aériennes, de cartes géopolitiques et de plans urbains, de bandes dessinées ou de tables de "Kriegspiel »; et encore des travellings sur l'eau de la Seine ou de Venise notamment, mais également des plans de vie nocturne parisienne ou une danse indigène tahitienne ; enfin, des extraits des Enfants du Paradis et des Visiteurs du soir de Carné, d'Orphée de Cocteau, de La charge de la Brigade légère de Michael Curtiz, mais également de Le troisième homme de Carol Reed ou de La charge fantastique de Raoul Walsh ; enfin de La Flèche Noire de Robin des Bois, de films sur la Seconde guerre mondiale avec des batailles navales ou amphibies, d'un Zorro de piètre qualité ou encore du premier film de Debord même, Hurlements en faveur de Sade, le seul dont la voix off nous donne les références.

Ce collage hétéroclite, dont on a donné ci-dessus un échantillon non exhaustif, produit des effets de sens par la juxtaposition et l'ordre de ces images fixes et mobiles, mais surtout par le rapport variable entre ces images et le commentaire sonore off lu par Debord qui les accompagne sans relâche, à l'exception de quelques courts intermèdes musicaux composés de trois seuls thèmes, graves et lyriques (deux concerts de Couperin et un standard de jazz, Whisper not), de quelques bandes sonores extraites des films cités, et de longs silences.

En particulier, quelques coïncidences remarquables entre les images et le commentaire off (concernant le public du cinéma, Guy Debord lui-même, dont on voit des portraits photographiques, quelques uns de ses amis situationnistes, son film Hurlements en faveur de Sade, mais aussi Paris, Venise et Florence et leurs images ou plans) alertent le spectateur, qui approfondira d'autres types de rapports, symboliques ou ironiques, entre ces deux dimensions du film debordien.

16 Enfin, un dernier élément, paratextuel et inhérent à la structure du film, se révèle être essentiel à sa compréhension : il s'agit du palindrome qu'illustre à la fois le titre du film et sa conclusion, où la traditionnelle «Fin » est remplacée par le sous-titre « À reprendre depuis le début ». 
17 On voit d'emblée comment le dispositif imaginé par Debord empêche à la fois toute narration filmique traditionnelle et toute identification au sens habituel : une approche faussement documentaire, paraphrasant Vertov mais extrêmement violente, renvoie le spectateur à sa « misère de milieu cadre » en le dépossédant de tout, jusqu'aux images du film le concernant ${ }^{16}$, tandis que le rapport sémantique entre le discours et les plans relève essentiellement d'une monstration analogique, plutôt que d'un récit, et c'est précisément parce qu'il s'agit de la monstration illustrant un discours que, pour l'essentiel, les plans peuvent se succéder de façon discontinue, sans constituer en soi de séquence ni de récit dramatique.

L'esthétique de Debord est toute entière placée sous le signe du " détournement ", dont lui-même et Wolman, se réclamant de Lautréamont, définissent assez précisément la pratique dans un texte de 1956 intitulé Mode d'emploi du détournement.

Dans un contexte révolutionnaire, «l'héritage littéraire et artistique de l'humanité doit être utilisé à des fins de propagande partisane » en dépassant toute idée de scandale pour aller vers « la négation de la négation » : ainsi,

Il va de soi que l'on peut non seulement corriger une œuvre ou intégrer divers fragments d'œuvres périmées dans une nouvelle, mais encore changer le sens de ces fragments et truquer de toutes les manières que l'on jugera bonnes ce que les imbéciles s'obstinent à nommer des citations. [...] Il faut concevoir un stade parodique sérieux où l'accumulation d'éléments détournés, loin de vouloir susciter l'indignation ou le rire en se référant à la notion d'une œuvre originale, mais marquant au contraire notre indifférence pour un original vidé de sens et oublié, s'emploierait à rendre un certain sublime ${ }^{17}$.

Cette pratique est encore bien présente dans In girum..., bien qu'il faille en nuancer les modalités : tout d'abord il faudrait distinguer les détournements littéraires, insérés dans le « commentaire », des détournements proprement filmiques.

À propos des premiers, on peut dire en consultant la liste paratextuelle qu'en livre Debord $^{18}$, qu'ils accompagnent des citations plus habituelles, quoique volontairement encyclopédiques, et qu'ils ancrent son discours dans une langue éminemment littéraire non dépourvue d'une respiration toute classique, dans une mémoire commune de l'humanité, l'élevant à une rhétorique élégante et grave, polémique et ironique, virtuose en un mot, quitte à "l'épicer» rarement de termes techniques ou familiers plus modernes. Il semble que peu d'observateurs aient souligné la beauté de la langue de Guy Debord, dont In girum ... un est des exemples les plus frappants.

Pour ce qui est des détournements photographiques ou filmiques, il faudrait encore distinguer ceux qui accompagnent le commentaire off de ceux qui l'interrompent. Les premiers, de loin les plus nombreux, constituent en durée les trois quarts environ du film. Les seconds, qui interrompent le commentaire par leur bande sonore, acquièrent une importance d'autant plus grande qu'ils sont longs : mais Debord insiste sur la fonction de ces détournements dans In girum..., par rapport à son film précédent, La Société du spectacle :

Dans le film La Société du spectacle, les films (de fiction) détournés par moi ne sont donc pas pris comme des «illustrations» critiques d'un art de la société spectaculaire, contrairement aux documentaires et actualités par exemples. Ces films de fiction volés, étant étrangers à mon film mais transportés là, sont chargés, quel qu'ait pu être leur sens précédent, de représenter, au contraire, le renversement $d u$ « renversement artistique de la vie».

Derrière le spectacle, il y avait la vie réelle qui a été déportée au-delà de l'écran. J’ai 
prétendu « exproprier les expropriateurs ». [...]

Il y a un déplacement dans In girum..., qui tient à plusieurs importantes différences : j'ai tourné directement une partie des images, j'ai écrit directement le texte pour ce film, enfin le thème du film n'est pas le spectacle, mais au contraire la vie réelle ${ }^{19}[\ldots$ ] nette qu'In girum... est selon Debord lui-même le plus abouti de ses films, impression que nous partageons en le revoyant aujourd'hui, aussi bien de par ses caractéristiques filmiques que, paradoxalement, de par son récit lyrique et épique, sur lequel nous voudrions nous arrêter un instant.

Le titre fournit une importante clé de lecture, ou plutôt l'une des clés du labyrinthe auquel le spectateur est ramené par la fin du film avec l'inscription « À reprendre depuis le début », qui apparaît en sous-titre du dernier plan. Mais pour en revenir au palindrome initial, souligné par le graphisme de la séquence titre et le silence qui l'accompagne, il s'agit d'un jeu de mot latin, anonyme et un peu obscur, que Debord traduit avec un lyrisme existentiel remarquable par: "nous tournons en rond dans la nuit, et nous sommes dévorés par le feu ». troisième personne du pluriel, mais il est surtout cité et commenté en son milieu, et souligné par la paraphrase du début de la Divine Comédie : "À la moitié du chemin de la vraie vie, nous étions environnés d'une sombre mélancolie ». Il s'agit sans doute de l'un des éléments de l'œuvre par lequel semble émerger plus distinctement une structure, un élément à partir duquel prend forme une problématique narration: ainsi avant sa reprise centrale il a été question dans le "commentaire" off du public du cinéma, du cinéma luimême et de l'auteur du film, de façon relativement linéaire; de la même façon, après cette parenthèse centrale, débute la narration de la guerre avant-gardiste, de ses héros et de ses batailles, enfin de son issue fatale et des cendres et de l'eau, après le feu. Mais le moment ouvert par la paraphrase dantesque, et composée d'une série de citations littéraires enchevêtrées, elles aussi lyriques et existentielles, semble constituer précisément le centre de ce palindrome labyrinthique, encadré par l'évocation du lieu de la conspiration et du combat par le feu, Paris, et de son double spéculaire et aquatique, Venise.

Ce moment central est remarquable aussi en ce qu'il donne à voir enfin les portraits des protagonistes du film, leurs visages ou leur masques, en inversant le rapport jusque là dominant entre les images et le commentaire: c'est ici le discours qui est un collage intertextuel obscur et parabolique, tandis que les images inaugurent la narration du conflit en montrant la «vrai vie» du «nous» des avant-gardistes, car, comme on l'a rappelé, « la thèse du film n'est pas le spectacle mais au contraire la vie réelle ».

Il semble également possible de confirmer la fonction prééminente de ce « milieu » dans l'économie du film en revenant sur l'intertextualité extrême qui le caractérise, aussi bien pour les images que pour son commentaire audio off: après Dante, se sont 
successivement une quartine d'Omar Khayyàm, le Jules César de Shakespeare, Le dit de l'enfant sage d'Alcuin, le Panégyrique de saint Bernard de Bossuet, et enfin le palindrome anonyme qui donne son titre au film qui sont convoqués; puis encore longuement l'Ecclésiaste, et enfin les dernière parole du général Jackson cité par Hemingway, avant que le commentaire ne redevienne personnel. Les images ainsi commentées constituent également un labyrinthe ${ }^{20}$ de significations, où l'on retrouve la compagnie des amis au café, des joueurs d'Échecs, les portraits de Chtcheglov, Wolman, Fonta, de Marbaix, ainsi que ceux de Debord lui-même à vingt ans et de la femme qu'il aimait alors; ensuite, des travelling et panoramiques de Paris, une séquence de guerre, et des plans réitérés et accolés de Paris et de l'Arsenale à Venise, avant le long détournement du film de Raoul Walsh They died with their boots on, avec Errol Flynn, qui raconte comment

Le régiment de Custer, formé en cercle, reçoit à coups de carabine les assauts successifs des indiens qui le cernent. Ses combattants tombent l'un après l'autre. À la fin les indiens submergent la position et exterminent les défenseurs ${ }^{21}$.

C'est ainsi que débute la troisième partie du film, progressivement annoncée encore par l'évocation du "passage rapide» de Chtcheglov, «celui qui ouvrit une route nouvelle, [qui] découvrit en un an des sujets de revendications pour des siècles, [dont] les profondeurs et les mystères de l'espace urbain furent [la] conquête ", et de son double détourné, le Prince Vaillant; c'est ainsi que débute le récit du combat mené par l'I.S. jusqu'à sa dissolution, rendue par le détournement positif de la charge de la Brigade légère : l'autre fin héroïque de ces happy few.

En règle générale, au cours du film, ce n'est pas l'image qui mène la narration, mais le commentaire en voix off, déclamé avec une infinie distanciation, et qui s'appuie sur les séquences visuelles par analogie ou en tire par contraste une force paradoxale, humoristique, ou violente. Pourtant, toujours à propos du rapport analogique entre le discours et les images, il est intéressant de remarquer qu'il finit par structurer lui aussi le film, en instaurant progressivement, par « reprises » croisées, un tissu symbolique tout bachelardien :

Tout le film est bâti sur le thème de l'eau. On y cite donc les poètes de l'écoulement de tout (Li Po, Omar Khayyâm; Héraclite, Bossuet, Shelley), qui tous ont parlé de l'eau: c'est le temps.

Il y a, secondairement, le thème du feu; de l'éclat de l'instant: c'est la révolution de Saint Germain des Prés, la jeunesse, l'amour, la négation dans sa nuit, le Diable, la bataille et les "entreprises inachevées" où vont mourir les hommes, éblouis en tant que "voyageurs qui passent"; et le désir dans cette nuit du monde ("nocte consumimur igni »).

Mais l'eau du temps demeure qui emporte le feu, et l'éteint. Ainsi l'éclatante jeunesse de Saint-Germain-des-Prés, le feu de l'assaut de l'ardente «Brigade légère » ont été noyés dans l'eau courante du siècle quand elles se sont avancées sous le canon du temps ${ }^{22} \ldots$

Cette compréhension progressive du symbole apparaît dans les images par la répétition d'une série de photogrammes et par la réitération de détournements filmiques:

[...] Il reste que les films qui interrompent le discours viennent plutôt le soutenir positivement, même s'il y a une certaine dimension ironique (Lacenaire, le Diable, le fragment de Cocteau, ou l'anéantissement du régiment de Custer). La Charge de la Brigade Légère veut «représenter ", très lourdement et élogieusement, une dizaine d'année de l' I.S ${ }^{23 !}$

Ce dernier exemple de lien symbolique semble particulièrement révélateur du rapport que Debord instaure entre son discours et ses images, un rapport qui ne relève plus tant 
alors de la discrépance, mais plutôt d'une analogie discours/image très variable, parfois distante, complexe ou révoltante, parfois plus facilement comique : et c'est ce rapport analogique réitéré et son organisation qui vont permettre de réintroduire la narration dans un film qui relevait dans sa première partie plutôt d'une volonté de monstration et de démonstration: "examen historique, théorie, essai, mémoires", selon les propres termes de son discours.

Mais La Charge de la Brigade Légère (The Charge of the light Brigade, réalisé par Michael Curtiz en 1936) nous semble jouir par ailleurs d'un statut à part parmi les «films volés " par Debord au regard de son interprète, Errol Flynn, que l'on retrouvait précisément dans le détournement de l'autre combat de l'avant-garde, le " défensif »: La Charge Fantastique ( They died with their boots on, Raoul Walsh, 1941), et qui marque de ce fait In girum...d'une présence singulière. Ce même western avait d'ailleurs déjà été détourné dans $L a$ Société $d u$ Spectacle, à propos duquel Debord écrit que « le film américain sur la guerre de Sécession (sur Custer) veut évoquer toutes les luttes de classes du XIX ${ }^{\mathrm{e}}$ siècle ; et même leur avenir ${ }^{24}$ .». Ainsi, la figure héroïque d'Errol Flynn acquiert un statut d'exception dans l'œuvre cinématographique de Debord, comparable à celle d'Orson Welles lui-même, que l'on retrouve également dans ses deux films comme une présence fascinante et ambiguëe ${ }^{25}$. Dans In girum..., le Troisième Homme, le Diable, Lacenaire et Zorro - comme d'autres «bandits lettrés » ou comme un lévrier afghan récalcitrant - offrent à Debord autant d'autres masques adéquats à l'image du paria qu'il revendique polémiquement, et qu'il creusera par ailleurs. Mais c'est Eroll Flynn, dont la vie fut aussi aventureuse que les films, qui s'affirme ici comme le pendant de Vaillant ; le Roland, le Renaud ou le Roger du poème épique de l'Arioste :

[...] ceux qui nous exposent diverses pensées sur les révolutions s'abstiennent ordinairement de nous faire savoir comment ils ont vaincu.

Mais moi, n'ayant pas ressemblé à tous ceux-là, je pourrai seulement dire, à mon tour, « les dames, les cavaliers, les armes, les amours, les conversations et les audacieuses entreprises » d'une époque singulière ${ }^{26}$.

31 Pourtant, en plus de ces aspects palindromique et symboliques, de son appartenance aux genres panégyrique, lyrique et épique, et précisément par-delà eux, dans sa dimension propagandiste et polémologique, dans son expérimentation et son exemplarité normative, dans sa transfiguration du vécu et sa mythogenèse, dans son invention formelle et conceptuelle, et sa dureté violente et zarathoustrienne, dans son encyclopédisme et dans sa promesse libertaire d'une "autonomie sans frein et sans règles ", In girum... nous semble relever d'un autre genre littéraire encore, spécifique aux avant-gardes et fer de lance de chacune d'entre elles, le manifeste. S'il est vrai que Guy Debord fut un avant-gardiste, et que ses « œuvres» se réfutent en tant que telles pour s'affirmer comme autant de manifestes surprenants, il n'est pas moins vrai que, "se disant cinéaste ", seul l'un de ses films pouvait porter si haut cet étendard de la guerre, du non retour et de la non réconciliation : ce film, c'est In girum imus nocte et consumimur igni, le tardif, le dernier, le plus mélancolique et impitoyable manifeste des avant-gardes par lequel tout est, dans la lagune palingénétique, « à reprendre depuis le début ${ }^{27}$ ». 


\section{ANNEXES}

Fiche technique

In girum imus nocte et consumimur igni (mars 1978)

Long métrage de $105 \mathrm{~min}$, format $35 \mathrm{~mm}$, noir et blanc.

Tourné en 1977. Produit par SIMAR film.

Ecrit et réalisé par Guy Debord.

Assistants réalisateurs : Elisabeth Gruet et Jean-Jacques Raspaud.

Chef opérateur : André Mrugalski.

Assistant opérateur : Richard Copans.

Montage : Stéphanie Granel, assistée de Christine Noël.

Ingénieur du son, mixage : Dominique Dalmasso.

Bruitage : Jérôme Lévy.

Documentaliste : Joëlle Barjolin.

Machiniste : Bernard Largemain.

Musique :

François Couperin :

- Prélude du Quatrième concert royal

- Premier mouvement du Nouveau concert nº ${ }^{\circ} 1$

Benny Golson :

- Whisper not (interprété par Art Blakey et les Jazz Messengers)

\section{NOTES}

1. Guy Debord, La Société du Spectacle, Paris, Champs Libre, 1971 (1967). On trouvera ce textes et les autres cités ici dans Guy Debord, CEuvres, Paris, Quarto Gallimard, 2006.

2. Cf. notamment le Traité de bave et d'éternité de Jean-Isidore Isou, 1950, et L'anticoncept de Gil J. Wolman, 1952.

3. Cf. Internationale Lettriste $n^{\circ} 1$, novembre 1952, in Guy Debord, Euvres, cit., p. 84.

4. Guy Debord, Contre le cinéma, Aarus, Institut scandinave de vandalisme comparé, 1964.

5. Olivier Assayas, Autour des films (documents), in Guy Debord, Euvres cinématographiques complètes, Paris, édition DVD Gaumont, aux soins d' A. Debord et O. Assayas, 2005. On ne peut que saluer le travail accompli par les éditeurs de ce remarquable coffret, et les en remercier, tout comme nous souhaitions remercier M. Patrick Marcolini pour ses conseils précieux.

6. Guy Debord, Rapport sur la construction des situations, in Euvres, cit. , p. 308.

7. Cf. la fiche technique du film, en annexe, ibid., p. 1410. 
8. Guy Debord, Des contrats, Cognac, Le Temps qu'il fait, 1995.

9. Guy Debord, Ordures et décombres déballés à la sortie du film In girum imus nocte et longuement igni par différentes sources autorisées, Paris, Champs Libre, 1982.

10. Guy Debord, In girum imus nocte et consumimur igni - Edition critique, Paris, Editions Gérard Lebovici, 1990.

11. On en trouvera confirmation dans les « Notes pour un contrat » du 7 janvier 1976, in Guy Debord, Autour des films, cit., p. 77.

12. Antoine Coppola, Introduction au cinéma de Guy Debord et de l'avant-garde situationniste, Arles, Sulliver, 2003.

13. Guy Debord, Panégyrique, Tome premier, Paris, Editions Gérard Lebovici, 1989; Panégyrique, Tome second, Paris, Fayard, 1997.

14. Guy Debord, Euvres, cit., p. 1421.

15. Guy Debord, In Girum imus nocte et longuement igni - Edition critique, in Euvres, cit., p. 1761.

16. Cf. le carton : " Ici les spectateurs, privés de tout, seront en outre privés d'images ", ibid., p. 1392.

17. Guy Debord et Gil J. Wolman, « Mode d'emploi du détournement », in Les lèvres nues $\mathrm{n}^{\circ}$ 8, Paris, mai 1956, cité in Euvres, cit. , p. 221.

18. Guy Debord, Euvres, cit. , p. 1413.

19. Guy Debord, « Notes sur l'emploi des films volés », ibid., p. 1411.

20. «C'était le labyrinthe le mieux fait pour retenir les voyageur » déclame Debord un peu plus tôt parlant de Paris. Ibid., p. 1366.

21. Cf. CEuvres, cit. p. 1375.

22. Note du 22 décembre 1977, in Guy Debord, Euvres, cit. , p. 1411.

23. Guy Debord, « Notes sur l'emploi des films volés », ibid. , p. 1411.

24. Guy Debord, Note sur l'emploi des films volés, in CEuvres cinématographiques complètes, cit. p. 1412.

25. M. Arkadin est détourné dans La Société du Spectacle, Le troisième homme dans In girum... On sait par ailleurs la grande admiration que vouait Debord à Welles.

26. Guy Debord, In girum imus nocte et consumimur igni, in Euvres, cit., p. 1355. Debord traduit significativement cortesie par 'conversations'.

27. Guy Debord, In girum imus nocte et longuement igni - Edition critique, in Euvres, cit., note de fin, p. 1789. "S'opposant aux traditionnelles marques de conclusion, "Fin" ou "A suivre", la phrase doit être comprise à tous les sens du verbe "reprendre". Elle veut dire d'abord que le film, dont le titre était un palindrome, eût gagné à être revu à l'instant, pour atteindre plus pleinement son effet désespérant : c'est quand on a connu la fin qu'on peut savoir comment il fallait comprendre le début. Elle veut dire aussi qu'il faudra recommencer, tant l'action évoquée que les commentaires à ce propos. Elle veut dire enfin qu'il faudra tout reconsidérer depuis le début, corriger, blâmer peut-être, pour arriver un jour à des résultats plus dignes d'admiration. » 


\section{RÉSUMÉS}

Vingt et un ans après le Rapport sur la construction des situations de 1957, qu'on peut considérer comme le manifeste fondateur de l'Internationale Situationniste, Guy Debord, "se disant cinéaste ", a tourné en 1978 son dernier long métrage, In girum imus nocte et consumimur igni, bilan tardif et mélancolique de cette même avant-garde. L'article analyse brièvement ce film, revenant sur certains de ses aspects formels et s'intéressant au récit qui y est paradoxalement réintroduit en même temps qu'une forme surprenante d'identification, qui renvoient au spectacle entendu comme aliénation narrative toujours refusé par Debord. Au demeurant, ce qui a été justement décrit comme un panégyrique se révèle être également, sous certains rapports, le dernier manifeste de l'I.S., voire du phénomène avant-gardiste qui a marqué de son sceau les arts du siècle passé.

\section{INDEX}

Mots-clés : avant-garde, Debord Guy, Internationale Situationniste, manifeste, palindrome, discrépance, détournement, identification, paratexte

Index chronologique : XXe siècle

\section{AUTEUR}

\section{SERGE MILAN}

Université de Nice-Sophia Antipolis, CIRCPLES, EA 3159 